\title{
miR-330-3p suppresses liver cancer cell migration by targeting MAP2K1
}

\author{
ZHE JIN, BAOXING JIA, LUDONG TAN and YAHUI LIU \\ Department of Hepatobiliary and Pancreatic Surgery, \\ The First Affiliated Hospital of Jilin University, Changchun, Jilin 130021, P.R. China
}

Received September 17, 2016; Accepted February 8, 2018

DOI: $10.3892 / \mathrm{ol} .2019 .10280$

\begin{abstract}
MicroRNAs, considered as a promising focus for the treatment of tumors, are key regulators of a large number of genes. The aim of the present study was to investigate the biological functions of microRNA (miR)-330-3p in liver cancer as it had been identified previously that miR-330-3p was deregulated in liver cancer. In order to identify the function of miR-330-3p in liver cancer, the expression of miR-330-3p was determined in liver cancer tissues and adjacent non-tumor tissues using reverse transcription-quantitative polymerase chain reaction analysis. To elucidate the function of miR-330-3p in liver cancer, miR-330-3p was overexpressed using mimic transfection. Cell migration was inhibited by miR-330-3p in liver cancer cells. The miRNA target prediction databases were used to identify potential target genes of miR-330-3p in liver cancer. The RNA level of mitogen-activated protein kinase kinase 1 (MAP2K1) was downregulated by miR-330-3p in liver cancer cells. In conclusion, miR-330-3p suppresses cell migration by targeting MAP2K1 in liver cancer cells.
\end{abstract}

\section{Introduction}

Liver cancer is the fifth most common type of cancer and the third leading cause of tumor-associated mortality worldwide, being particularly prevalent in Asia (1). The most effective treatment for liver cancer is currently surgery (2). However, the characteristics of liver cancer, including multifocal development or distant metastasis, preclude surgical treatment for the patients from being curative (3). Therefore, in order to determine the biology of liver cancer, it is important to gain a thorough understanding of the underlying molecular mechanisms of tumor growth and metastasis.

Correspondence to: Dr Yahui Liu, Department of Hepatobiliary and Pancreatic Surgery, The First Affiliated Hospital of Jilin University, 71 Xinmin Street, Changchun, Jilin 130021, P.R. China E-mail: liuyahui_jilin@163.com

Key words: liver cancer, microRNA, mitogen-activated protein kinase kinase 1, migration, reverse transcription-quantitative polymerase chain reaction
Mature microRNAs (miRNAs) are a group of short non-coding RNAs containing between 18 and 26 nucleotides, which, at the post-transcriptional level, regulate target gene expression by targeting the $5^{\prime}$ or $3^{\prime}$-untranslated regions (UTRs) of mRNA (4). miRNAs regulate a number of biological processes, and their dysregulation has been demonstrated to be associated with development, proliferation, stress resistance, metastasis and apoptosis of cancer cells by regulating oncogenes or tumor suppressors (5), including in liver cancer. For example, microRNA (miR)-224 promotes liver cancer proliferation and metastasis (6); conversely, miR-335 (7) and miR-424 (8) suppress liver cancer proliferation and metastasis. Furthermore, miR-330-3p has been identified to be deregulated in malignant liver cancer (9). However, the association of miR-330-3p with liver cancer and its function in liver cancer remains unclear.

In the present study, the function of miR-330-3p in liver cancer was investigated, with the aim of providing new insights into the pathology of liver cancer, which may have value in the development of diagnostics and therapeutics for liver cancer.

\section{Materials and methods}

Patient samples. In total, 30 cases of liver cancer samples were collected between May 2012 and April 2015 at The First Hospital of Jilin University (Changchun, China). Prior to surgery, none of the 30 patients received chemotherapy or radiation therapy. Prior to use, the tissue samples were stored in liquid nitrogen. The present study was approved by the ethical committee of the First Affiliated Hospital of Jilin University. Table I presents information concerning patient age, sex, and cancer stage were obtained from patient records.

Cell culture and treatment. The human liver cancer cell line HepG2 were obtained from the American Type Culture Collection (Manassas, VA, USA). HepG2 cells were maintained in Dulbecco's modified Eagle's medium (DMEM; Invitrogen; Thermo Fisher Scientific, Inc., Waltham, MA, USA) supplemented with $10 \%$ fetal bovine serum (FBS; HyClone; GE Healthcare, Logan, UT, USA) at $37^{\circ} \mathrm{C}$ in a humidified atmosphere containing 5\% $\mathrm{CO}_{2}$. miR-330-3p mimic (5'-CTG CAGAGAGGCAGCGCTGT-3') and negative control (NC) (termed as NC, 5'-ACUACUGAGUGACAGUAGA-3') oligonucleotides were purchased from Guangzhou RiboBio Co., 
Ltd. (Guangzhou, China). Transfection of cells with $50 \mathrm{nM}$ oligonucleotides was carried out using Lipofectamine ${ }^{\circledR} 2000$ (Invitrogen; Thermo Fisher Scientific, Inc.), according to the manufacturer's protocol.

Target gene prediction and verification. The target genes of miR-330-3p were predicted using three bioinformatics algorithms: PicTar (https://pictar.mdc-berlin.de), miRbase (http://www.mirbase.org) and TargetScan (http://www. targetscan.org). Mitogen-activated protein kinase kinase 1 (MAP2K1) was identified to be downregulated by miR-330-3p, therefore, from human genomic DNA, the full-length 3'UTR of MAP2K1 was amplified and cloned into pMIR-GLOTM luciferase vector (Promega Corporation, Madison, WI, USA) to serve as the wild-type. The mutations were achieved using site-directed mutagenesis QuickChange ${ }^{\mathrm{TM}}$ Multi Site-Directed Mutagenesis kit (Clontech Laboratories, Inc., Mountainview, CA, USA). A total of $1 \times 10^{4} \mathrm{HepG} 2$ cells was plated in 48-well plates, and co-transfection was performed using $200 \mathrm{ng}$ wild-type, mutant or pMIR-GLOTM empty vector, and either $80 \mathrm{ng}$ miR-330-3p expression vector or $80 \mathrm{ng}$ pcDNA3.1(+) empty vector. At $48 \mathrm{~h}$ after transfection, cells were harvested and were assayed for firefly and Renilla luciferase activity using a Dual-Luciferase Glow assay kit (Promega Corporation) according to the manufacturer's protocol. Overexpression of MAP2K1 was achieved using a pCMV-MAP2K1 expression plasmid. All transfection experiments were conducted in triplicate.

Wound healing assay. For the wound-healing assay, HepG2 cells were collected and trypsinized, followed by seeding equally in 6-well cell culture plates. After $24 \mathrm{~h}$, the cells reached confluence. Using a sterile $100 \mu 1$ pipette tip, artificial homogeneous wounds in the monolayer were created. The cell culture plate was washed with serum-free DMEM. The wound distance was determined at 0 and $24 \mathrm{~h}$ under a reflected-light microscope (magnification, $\mathrm{x} 40$ ).

Cell migration assay. To determine cell invasion capability, a Transwell assay was used. Prior to the experiment, HepG2 cells were transfected with miR-330-3p mimic or NC oligonucleotides. After $16 \mathrm{~h}$, transfected cells were typsinized and resuspended in DMEM with 10\% FBS (Life Technologies; Thermo Fisher Scientific, Inc.), and $1.0 \times 10^{4}$ cells were placed into the upper chambers $(8-\mu \mathrm{m}$ pore size; EMD Millipore, Billerica, MA, USA) with $200 \mu$ l RPMI-1640 medium. The lower chambers were filled with $600 \mu 1$ DMEM supplemented with $10 \%$ FBS. Following incubation at $37^{\circ} \mathrm{C}$ for $48 \mathrm{~h}$, non-invading cells were removed from the top of the chamber. The migratory cells in the lower chamber were fixed with $90 \%$ methyl alcohol at room temperature for $30 \mathrm{~min}$ and stained with $0.1 \%$ crystal violet. Stained cells in five randomly selected fields were counted under a microscope (magnification, x200).

To verify the function of MAP2K1 in the suppression of liver cancer cell migration by miR-330-3p, HepG2 cells were transfected with miR-330-3p together with the MAP2K1 expression vector pCMV-MAP2K1. For lentivirus-mediated expression of SNF2H, FLAG-tagged full-length SNF2H ORF was cloned in pWPI.1 (plasmid no. 12254; Addgene, Inc., Cambridge, MA, USA). For RNA interference of SNF2H expression, DNA fragments encoding the hairpin precursors for shSNF2H\#1 (5'-CGTCGAATTAAGGCTGATGTT-3') and shSNF2H\#2 (5'-CGACTGCTGATGTAGTAATTT-3') were inserted into the pLKO.1-TRC cloning vector. A scrambled small interfering RNA precursor (Scr) of similar GC-content to shSNF2H\#1 and shSNF2H\#2, but without sequence identity to SNF2H cDNA, was used as the control. The MAP2K1 expression vector pCMV-MAP2K1 and miR-330-3p mimic were co-transfected into HepG2 cell using Lipofectamine 2000 (Thermo Fisher Scientific, Inc.). After 48 h, HepG2 cells were collected for the following experiments.

Western blot analysis. HepG2 cells were transfected with miR-330-3p mimic or NC oligonucleotides and incubated at $37^{\circ} \mathrm{C}$ for $24 \mathrm{~h}$. Total protein was isolated from cell pellets with radioimmunoprecipitation lysis buffer supplemented with Complete protease and phosphatase inhibitor cocktail (Roche Diagnostics, Basel, Switzerland). Proteins (20 $\mu \mathrm{g})$ were separated by $12 \%$ SDS-PAGE. Electrophoretically separated proteins were transferred onto polyvinylidene difluoride membranes (Thermo Fisher Scientific, Inc.). Membranes were blocked with $5 \%$ non-fat milk at room temperature for $2 \mathrm{~h}$. Blots were probed for MAP2K1 (1:10,000, cat. no. ab32134; Abcam) and $\beta$-actin loading control (1:5,000 cat. no. ab8227; Abcam). Antibodies bound to target proteins were visualized using the Enhanced Chemiluminescence Western Blotting Detection Reagent (Santa Cruz Biotechnology, Dallas, TX, USA). Antibodies bound to target proteins were visualized using the Enhanced Chemiluminescence Western Blotting Detection Reagent (Santa Cruz Biotechnology, Dallas, TX, USA). The images were quantified using the LAS3000 imaging system (Fujifilm Corporation, Tokyo, Japan).

RNA isolation and reverse transcription-quantitative polymerase chain reaction ( $R T-q P C R)$. HepG 2 cells transfected with miR-330-3p mimic or NC oligonucleotides were incubated for $24 \mathrm{~h}$ and collected using the mirVana Kit (Ambion Inc., Austin, TX, USA), total RNA was isolated. cDNA was generated using a PrimeScript ${ }^{\mathrm{TM}}$ RT-qPCR kit from total RNA and amplified according to the manufacturer's protocol (Takara Biotechnology Co., Ltd., Dalian, China). Amplification was performed with the following thermocycling conditions: $5 \mathrm{~min}$ at $95^{\circ} \mathrm{C}$, followed by 40 cycles of $95^{\circ} \mathrm{C}$ for $30 \mathrm{sec}$ and $65^{\circ} \mathrm{C}$ for $45 \mathrm{sec}$. Primer sequences are presented in Table II. The expression of the target gene or miRNA was normalized to GAPDH or U6, respectively. Analysis of relative gene expression data was performed using the real-time quantitative PCR and the $2^{-\Delta \Delta \mathrm{Cq}}$ method (10).

Statistical analysis. Results are expressed as the mean \pm standard deviation. Each experiment was repeated at least three times. SPSS (version 13.0; SPSS, Inc., Chicago, IL, USA) was used for statistical analysis. The statistical significance of the difference between groups was determined by analysis of variance (two-way ANOVA) with Duncan's post-hoc test) or an unpaired Student's t-test. $\mathrm{P} \leq 0.05$ was considered to indicate a statistically significant difference.

\section{Results}

miR-330-3p is downregulated in patients with liver cancer. In order to determine the expression level of miR-330-3p in 
Table I. Clinicopathological characteristics of patients with liver cancer and healthy volunteers.

Patients with liver cancer $(\mathrm{n}=30)$

Characteristic

$\operatorname{BCLC} 0(n=3) \quad \operatorname{BCLC} A(n=12) \quad \operatorname{BCLC~B~}(n=5) \quad$ BCLC C+D $(n=10)$

Normal $(n=20)$

\section{Clinical factors}

Age, years

Sex (male/female)

AST, IU/1

$\begin{array}{cc}61.2 \pm 9.7 & 56.2 \pm 8.8 \\ 2 / 1 & 10 / 2 \\ 48.6 \pm 35.7 & 56.1 \pm 78.2 \\ 51.8 \pm 47.2 & 54.8 \pm 83.3 \\ 3 / 0 & 11 / 1\end{array}$
$56.9 \pm 8.4$
$1 / 4$

ALT, IU/1

Child-pugh classification

$11 / 1$

\section{$54.5 \pm 44.6$}

$49.4 \pm 33.1$

$4 / 1$
$52.5 \pm 11.2$
$8 / 2$
$67.8 \pm 49.3$

$54.4 \pm 12.4$

$14 / 6$

$11.6 \pm 5.4$

(A/B) (23)

Tumor-associated factors

$$
\text { AFP, ng/ml }
$$

$148.0 \pm 378.3$

$$
256.5 \pm 426.6
$$

$254.4 \pm 405.2$

$789.5 \pm 628.7$

$6.39 \pm 5.85$

Tumor number

(single/multiple)

Tumor size, $\mathrm{cm}^{3}$

Vascular invasion (-/+)

$3 / 0$

$11 / 1$

$2.1 \pm 0.5$

$3.4 \pm 1.2$

$1 / 4$

$3 / 0$

$12 / 0$

$6.1 \pm 2.3$

$4 / 6$

$0 / 1 / 2$

$1 / 9 / 2$

$5 / 0$

$7.5 \pm 2.7$

Edmondson grade (I/II/III) (24)

$1 / 2 / 2$

$3 / 7$

$1 / 7 / 2$

BCLC, Barcelona Clinic Liver Cancer (25); AST, aspartate aminotransaminase; ALT, alanine aminotransferase; AFP, $\alpha$-fetoprotein.

Table II. Primer sequence for reverse transcription-quantitative polymerase chain reaction.

\begin{tabular}{lll}
\hline Gene & \multicolumn{1}{c}{ Sense primer (5'-3') } & Antisense primer (5'-3') \\
\hline U6 & TGCGGGTGCTCGCTTCGGCA & CCAGTGCAGGGTCCGAGGT \\
$\beta$-actin & CTCCATCCTGGCCTCGCTGT & GCTGTCACCTTCACCGTTCC \\
miR-330-3p & CAACTGCCTCTCTGGGCCTG & CTGCAGAGAGGCAGCGCTG \\
MAP2K1 & CAAGAAGAAGCCGACGCCCAT & GACGCCAGCAGCATGGGTTG
\end{tabular}

miR, microRNA; MAP2K1, mitogen-activated protein kinase kinase 1.
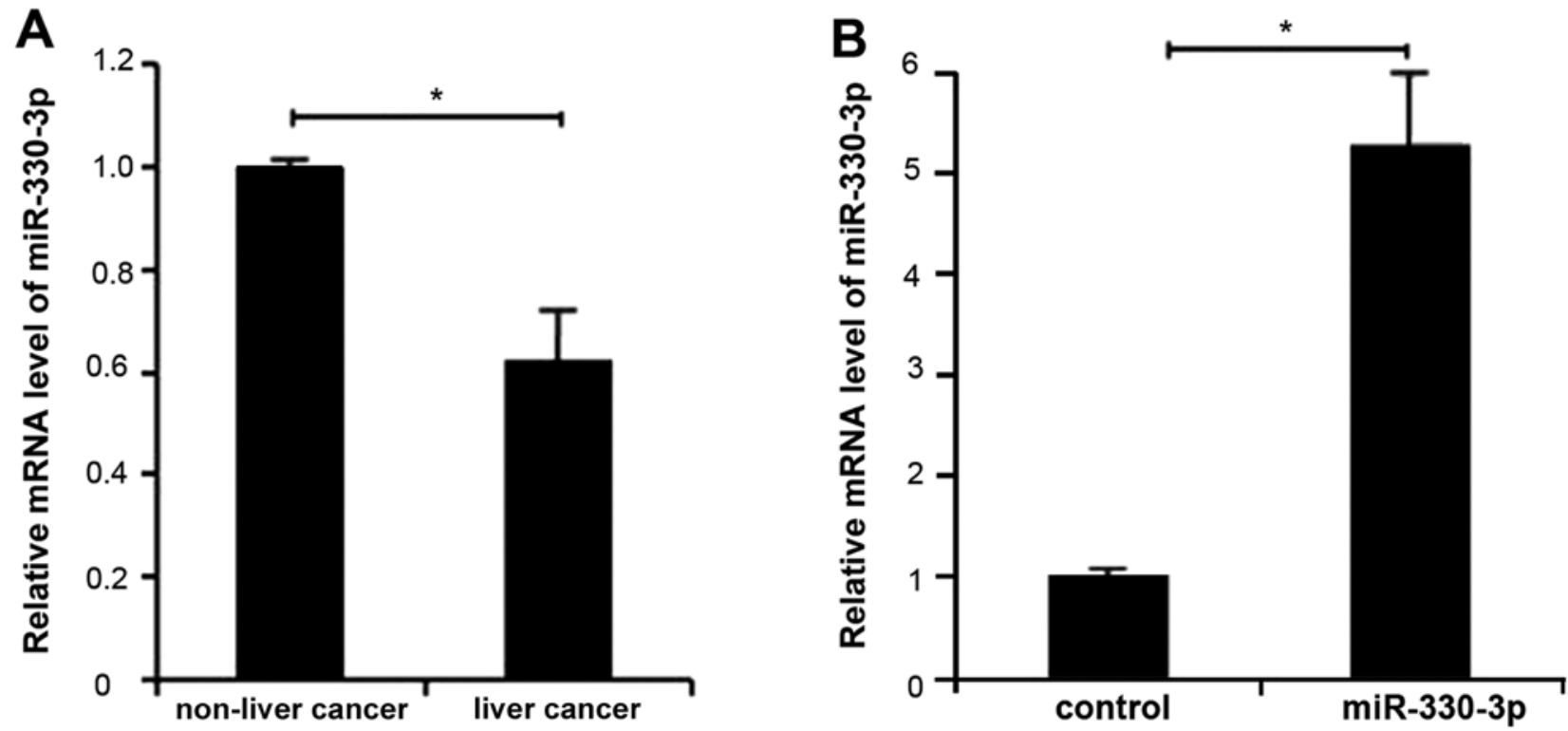

Figure 1. (A) Detection of miR-330-3p expression in liver cancer tissue or non-tumor tissue using the reverse transcription-quantitative polymerase chain reaction. (B) miR-330-3p expression was increased by transfection of miR-330-3p mimic. miR, microRNA. Results are expressed as the mean \pm standard deviation for three independent experimental repeats. ${ }^{*} \mathrm{P}<0.05$. 

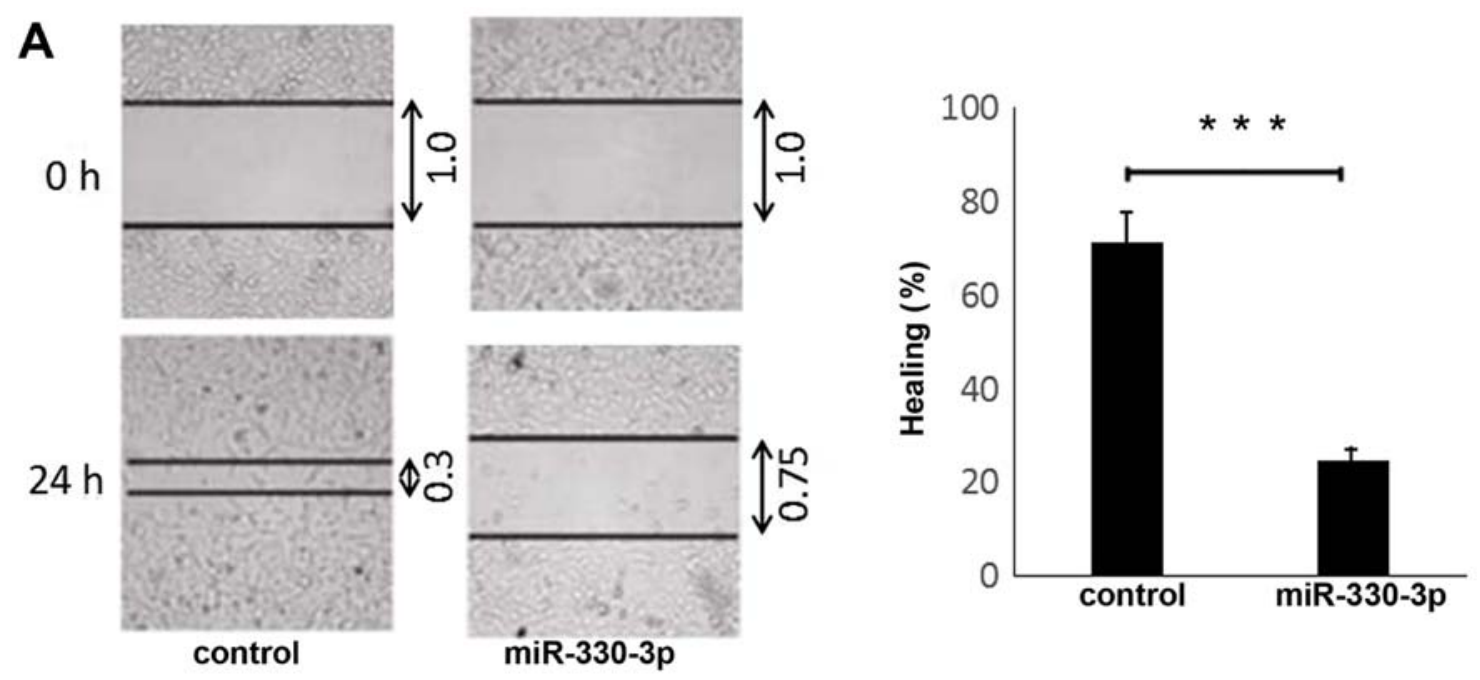

B
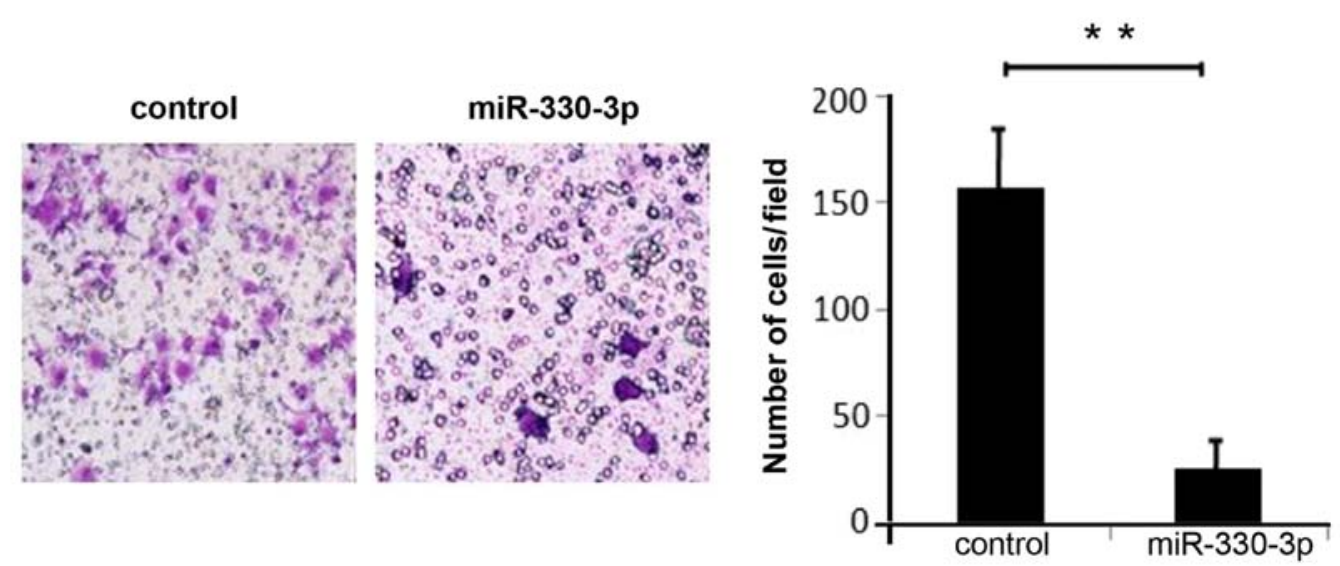

Figure 2. (A) Wound healing assay to determine the effect of miR-330-3p on cell migration. (B) A Transwell assay to determine the effect of miR-330-3p on cell migration. Results are expressed as the mean \pm standard deviation for three independent experiments. miR, microRNA. ${ }^{* *} \mathrm{P}<0.01,{ }^{* * *} \mathrm{P}<0.001$.

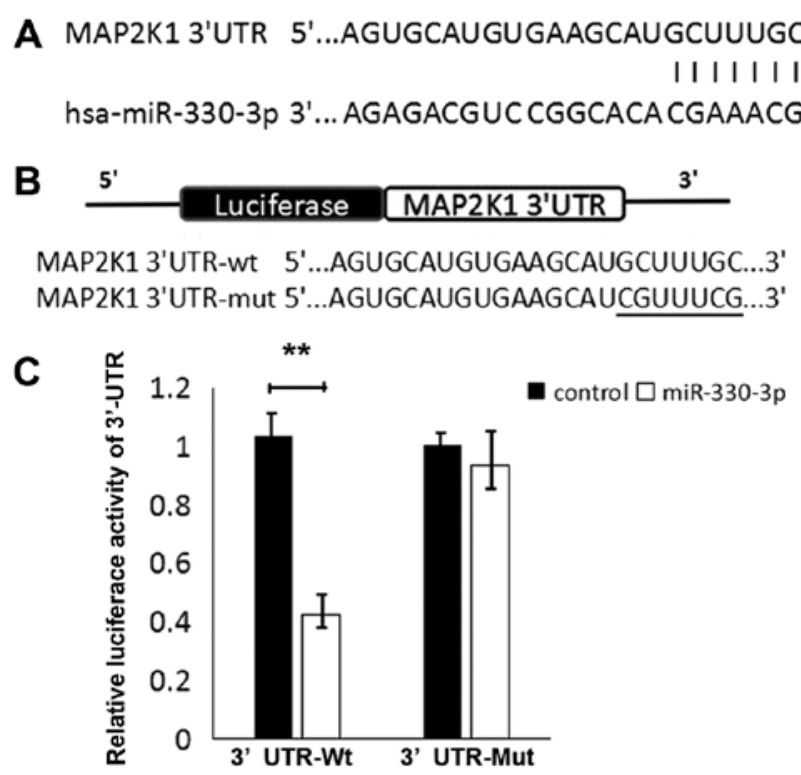

Figure 3. (A) Complementarity between miR-330-3p and MAP2K1 sequences. (B) Construction of reporter gene plasmids and point mutation plasmids of MAP2K1 3'UTR. (C) A luciferase reporter assay results demonstrated a significant decrease in the activity of wild-type gene plasmid MAP2K1 3'UTR, but no effect on mutant plasmids. Results are expressed as the mean \pm standard deviation. ${ }^{* *} \mathrm{P}<0.01 . \mathrm{miR}$, microRNA; MAP2K1, mitogen-activated protein kinase kinase 1; UTR, untranslated region; hsa, human; Wt, wild-type; Mut, mutant. liver cancer tissue, mRNAs were extracted from liver cancer tissues and analyzed using RT-qPCR. Compared with normal tissues, the expression of miR-330-3p in liver cancer tissues was significantly decreased $(\mathrm{P}<0.05$; Fig. 1A). The results suggested that deregulation of miR-330-3p was associated with the progression of liver cancer.

miR-330-3p suppresses migration of liver cancer cells. miR-330-3p expression was significantly upregulated in HepG2 cells by transfection of miR-330-3p mimic $(\mathrm{P}<0.05$; Fig. 1B). As observed in wound healing assays, cell migration was inhibited by the expression of miR-330-3p in HepG2 cells $(\mathrm{P}<0.01$; Fig. 2A). Transwell assays confirmed that overexpression of miR-330-3p significantly inhibited liver cancer cell migration $(\mathrm{P}<0.05$; Fig. $2 \mathrm{~B})$.

MAP2K1 is post-transcriptionally downregulated by $m i R-330-3 p$. To investigate the underlying mechanism by which miR-330-3p inhibits liver cancer cell migration, potential targets of miR-330-3p were identified. As predicted using TargetScan, PicTar and miRanda, MAP2K1 was selected as the candidate for further research owing to its vital functions in tumorigenesis and cancer progression. Wild-type and mutant MAP2K1 3'UTRs were constructed and it was identified that miR-330-3p significantly decreased luciferase activity 

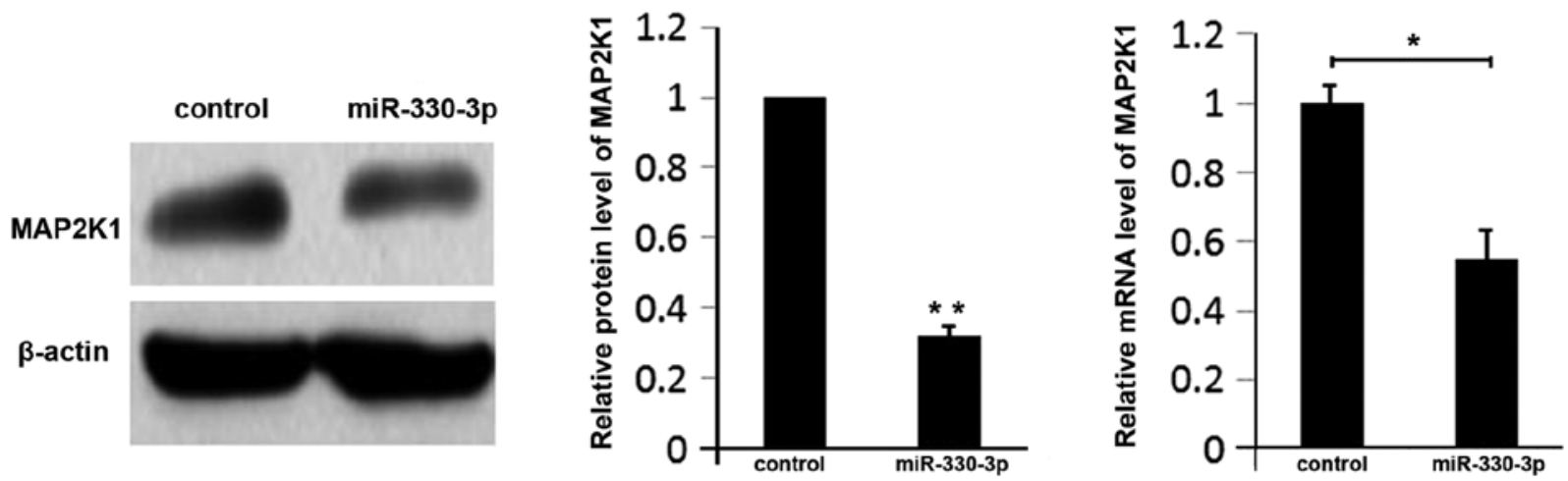

Figure 4. HepG2 cells were transfected using miR-330-3p mimic or negative control, prior to determining MAP2K1 mRNA and protein expression. Results are expressed as the mean \pm standard deviation. ${ }^{*} \mathrm{P}<0.05$. miR, microRNA; MAP2K1, mitogen-activated protein kinase kinase $1 .{ }^{*} \mathrm{P}<0.05,{ }^{* *} \mathrm{P}<0.01$.
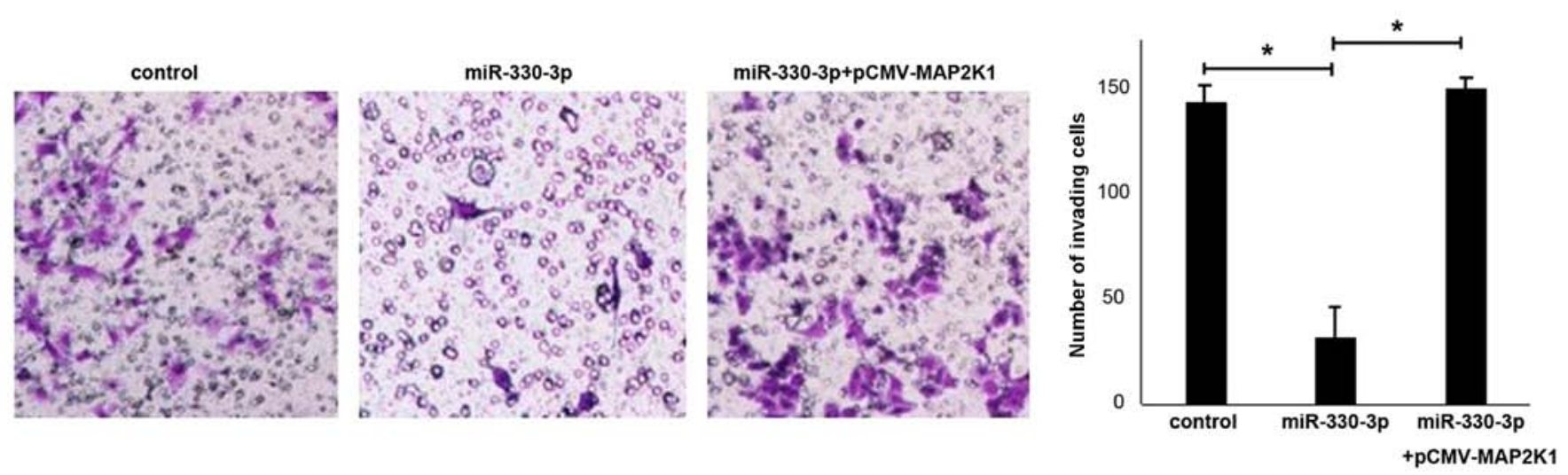

Figure 5. Migratory ability of HepG2 cells with the indicated transfections were determined using Transwell assays. Results are expressed as the mean \pm standard deviation. ${ }^{*} \mathrm{P}<0.05$. miR, microRNA.

in HepG2 cells transfected with wild-type MAP2K1 3'UTR $(\mathrm{P}<0.01$; Fig. 3), which was not observed following transfection with mutant 3'UTR.

Conversely, overexpression of miR-330-3p in liver cancer cells inhibited the expression of MAP2K1 at the mRNA and protein levels (Fig. 4). These results suggested that miR-330-3p regulates the expression of MAP2K1 in liver cancer.

miR-330-3p inhibits liver cancer cell migration by downregulating $M A P 2 K 1$. To verify the function of MAP2K1 in the suppression of liver cancer cell migration by miR-330-3p, HepG2 cells were transfected with miR-330-3p together with the MAP2K1 expression vector pCMV-MAP2K1. Compared with the control group, an increased number of liver cancer cells transfected with miR-330-3p lost the ability of migration, while cells co-transfected with pCMV-MAP2K1 exhibited increased migration abilities in the Transwell assay (Fig. 5). The results suggested that the increased expression of MAP2K1 contributed to the migratory ability of HepG2 cells.

\section{Discussion}

Invasion typically leads to metastasis and these events are primary reasons for the poor prognosis of liver cancer (11). For liver cancer, surgical resection is the most effective therapy, but only between 10 and $15 \%$ of patients are suitable for surgery; the majority of the remaining patients exhibit distant metastases at diagnosis, which renders curing liver cancer difficult $(11,12)$. Therefore, it is important to identifyfactors vital in invasion and metastasis and the underlying mechanisms. Investigation into miRNAs has led to novel studies to identify the molecular mechanism of cancer cell metastasis $(13,14)$, and therefore the development of potential therapeutics in the treatment of human cancer.

Previous results support a fundamental function of microRNAs in tumor invasion and metastasis; these biological functions are associated with the deregulation of microRNAs in various types of cancer (14). Previous studies have suggested that miR-330-3pis deregulated in the tissues and blood of patients with non-small-cell lung cancer (15). In the present study, it was identified that miR-330-3p was downregulated in patients with liver cancer. Furthermore, miR-330-3p regulates the proliferation and migration of prostate cancer cells (16). These results prompted the investigation of whether miR-330-3p functioned in a similar manner in liver cancer. Therefore, miR-330-3p was restored in liver cancer cells by high efficiency transfection. The results revealed that the migration of liver cancer cells was inhibited by miR-330-3p. By combining the 3'UTRs of target genes, miRNAs were able to regulate many genes. Studies to identify miRNA target genes have identified several novel cancer invasion and metastasis suppressor genes. Identification of new targets involved 
in the metastasis of liver cancer may lead to novel diagnostic strategies or drug development.

Mitogen-activated protein kinase/extracellular-signal-regulated kinase activation has been reported to be associated with the malignant progression of liver cancer. Overexpression of activated MAP2K1 enhances cancer cell proliferation and confers drug resistance on the cells (17). Using bioinformatic analysis, MAP2K1 was identified as one of the targets of miR-330-3p. It was also observed that upregulation of miR-330-3p in liver cancer cells led to downregulation of MAP2K1 at the RNA and protein levels. The negative regulation of MAP2K1 by miR-330-3p was demonstrated using a luciferase reporter assay. Furthermore, when MAP2K1 and miR-330-3p were knocked down, the migratory ability of HepG2 cells was also inhibited. These results suggested that the function of miR-330-3p in suppressing migration of liver cancer cells was mediated by MAP2K1.

As a number of miRNAs have been reported to be associated with the progression of liver cancer (18-20), those miRNAs may have synergistic or expanded effect on biological behavior of tumor cells. Compared with pure blocking reagent, miRNAs may have an advantage on influencing the tumor microenvironment $(21,22)$. In this regard, the results of the present study provide novel insights into the suppressor function of miR-330-3p and network of miRNAs in liver cancer. The focus of future studies will be on the effect of miR-330-3p in vivo.

\section{Acknowledgements}

Not applicable.

\section{Funding}

No funding was received.

\section{Availability of data and materials}

The datasets used and/or analyzed during the current study are available from the corresponding author on reasonable request.

\section{Authors' contribution}

ZJ designed the study, analyzed the data and wrote the manuscript. BJ and LT performed the experiments and prepared the figures. LT and YL collected the clinical specimens and analyzed the clinical data. All authors reviewed the manuscript.

\section{Ethics approval and consent to participate}

All protocols dealing with the patients conformed to the ethical guidelines of the Helsinki Declaration and were approved by the ethics committee of First affiliated Hospital of Jilin University. Patients provided written informed consent.

\section{Patient consent for publication}

Patients provided consent for the publication of any data and associated images.

\section{Competing interests}

The authors declare that they have no competing interests.

\section{References}

1. Ferlay J, Shin HR, Bray F, Forman D, Mathers C and Parkin DM: Estimates of worldwide burden of cancer in 2008: GLOBOCAN 2008. Int J Cancer 127: 2893-2917, 2010.

2. Forner A, Hessheimer AJ, Isabel Real M and Bruix J: Treatment of hepatocellular carcinoma. Crit Rev Oncol Hematol 60: 89-98, 2006.

3. Jeng KS and Ching HJ: The role of surgery in the management of unusual complications of transcatheter arterial embolization for hepatocellular carcinoma. World J Surg 12: 362-368, 1988.

4. Bartel DP: MicroRNAs: Target recognition and regulatory functions. Cell 136: 215-233, 2009.

5. Ryan BM, Robles AI and Harris CC: Genetic variation in microRNA networks: The implications for cancer research. Nat Rev Cancer 10: 389-402, 2010.

6. Ma D, Tao X, Gao F, Fan C and Wu D: miR-224 functions as an onco-miRNA in hepatocellular carcinoma cells by activating AKT signaling. Oncol Lett 4: 483-488, 2012.

7. Liu H, Li W, Chen C, Pei Y and Long X: MiR-335 acts as a potential tumor suppressor miRNA via downregulating ROCK1 expression in hepatocellular carcinoma. Tumour Biol 36: 6313-6319, 2015.

8. Zhang Y, Li T, Guo P, Kang J, Wei Q, Jia X, Zhao W, Huai W, Qiu Y, Sun L and Han L: MiR-424-5p reversed epithelial-mesenchymal transition of anchorage-independent HCC cells by directly targeting ICAT and suppressed HCC progression. Sci Rep 4: 6248, 2014

9. Chen J, Wu X, Cai B, Su Z, Li L, An Y and Wang L: Circulating microRNAs as potential biomarkers of HBV infection persistence. Infect Genet Evol 54: 152-157, 2017.

10. Kenneth $\mathrm{J}$ and Livak TD: Analysis of relative gene expression data using rea $1 \&$ mdash;time quantitative PCR a nd the $2 \square \mathrm{ct}$ method. Method, 2001.

11. Vitali GC, Laurent A, Terraz S, Majno P, Buchs NC, Rubbia-Brandt L, Luciani A, Calderaro J, Morel P, Azoulay D and Toso C: Minimally invasive surgery versus percutaneous radio frequency ablation for the treatment of single small $(</=3 \mathrm{~cm})$ hepatocellular carcinoma: A case-control study. Surg Endosc 30: 2301-2307, 2016.

12. Xiong W, Sun LP, Chen XM, Li HY, Huang SA and Jie SH: Comparison of microRNA expression profiles in HCC-derived microvesicles and the parental cells and evaluation of their roles in HCC. J Huazhong Univ Sci Technolog Med Sci 33: 346-352, 2013.

13. Deng S, Calin GA, Croce CM, Coukos G and Zhang L: Mechanisms of microRNA deregulation in human cancer. Cell Cycle 7: 2643-2646, 2008.

14. Dinkova-Kostova AT, Jenkins SN, Fahey JW, Ye L, Wehage SL, Liby KT, Stephenson KK, Wade KL and Talalay P: Protection against UV-light-induced skin carcinogenesis in SKH-1 high-risk mice by sulforaphane-containing broccoli sprout extracts Cancer Lett 240: 243-252, 2006.

15. Wei CH, Wu G, Cai Q, et al.: MicroRNA-330-3p promotes cell invasion and metastasis in non-small cell lung cancer through GRIA3 by activating MAPK/ERK signaling pathway. J Hematol Oncol 10: 125, 2017.

16. Mao Y, Chen H, Lin Y, Xu X, Hu Z, Zhu Y, Wu J, Xu X, Zheng X and Xie L: microRNA-330 inhibits cell motility by downregulating Sp1 in prostate cancer cells. Oncol Rep 30: 327-333, 2013.

17. Huynh H, Nguyen TT, Chow KH, Tan PH, Soo KC and Tran E: Over-expression of the mitogen-activated protein kinase (MAPK) kinase (MEK)-MAPK in hepatocellular carcinoma: Its role in tumor progression and apoptosis. BMC Gastroenterol 3: 19, 2003.

18. Liang HH, Wei PL, Hung CS, Wu CT, Wang W, Huang MT and Chang YJ: MicroRNA-200a/b influenced the therapeutic effects of curcumin in hepatocellular carcinoma (HCC) cells. Tumour Biol 34: 3209-3218, 2013.

19. Chen X, Zhang L, Zhang T, Hao M, Zhang X, Zhang J, Xie Q, Wang Y, Guo M, Zhuang $\mathrm{H}$ and Lu F: Methylation-mediated repression of microRNA 129-2 enhances oncogenic SOX4 expression in HCC. Liver Int 33: 476-486, 2013.

20. Zhang D, Zhou P, Wang W, Wang X, Li J, Sun X and Zhang L: MicroRNA- 616 promotes the migration, invasion and epithelial-mesenchymal transition of HCC by targeting PTEN. Oncol Rep 35: 366-374, 2016. 
21. Paiva I, Gil da Costa RM, Ribeiro J, Sousa H, Bastos MM, Faustino-Rocha A, Lopes C, Oliveira PA and Medeiros R: MicroRNA-21 expression and susceptibility to HPV-induced carcinogenesis-role of microenvironment in K14-HPV16 mice model. Life Sci 128: 8-14, 2015.
22. Paiva I, Gil da Costa RM, Ribeiro J, Sousa H, Bastos M, Faustino-Rocha A, Lopes C, Oliveira PA and Medeiros R: A role for microRNA-155 expression in microenvironment associated to HPV-induced carcinogenesis in K14-HPV16 transgenic mice. PLoS One 10: e0116868, 2015. 